\title{
Neue Option für signifikante Symptombesserung
}

Mit der Anfang des Jahres erfolgten Zulassung des oralen selektiven Phosphodiesterase 4 (PDE4)-Hemmers Apremilast durch die Europäische Kommission (EC) steht für Patienten mit Plaque-Psoriasis und für solche mit Psoriasis-Arthritis nun eine neue wirksame Alternative zur Verbesserung der Symptome zur Verfügung.

Apremilast wird für zwei Indikationen zugelassen: 1. Zur Behandlung mittelschwerer bis schwerer chronischer Plaque-Psoriasis bei erwachsenen Patienten, die auf eine andere systemische Therapie nicht angesprochen haben oder bei denen eine Kontraindikation oder Intoleranz gegenüber anderen systemischen Therapien wie Ciclosporin, Methotrexat oder Psoralen und UVALicht (PUVA) vorliegt. 2. als Monotherapie oder in Kombination mit krankheitsmodifizierenden Antirheumatika (DMARDs) zur Behandlung aktiver Psoriasis-Arthritis (PsA) bei erwachsenen Patienten, die auf eine frühere DMARD-Therapie unzureichend angesprochen oder diese nicht vertragen haben.

\section{Sicherheit und Wirksamkeit in ESTEEM und PALACE gezeigt}

Die Marktzulassung basiert auf den Sicherheits- und Wirksamkeitsergebnissen der beiden Phase III Programme ESTEEM und PALACE, bei denen ein anhaltendes klinisches Ansprechen von Patienten mit Psoriasis (ESTEEM) und Psoriasis-Arthritis (PALACE) bei der Behandlung mit Apremilast über einen Zeitraum von 52 Wochen bei zahlreichen Endpunkten nachgewiesen wurde.

Im ESTEEM-Studienprogramm führte die Behandlung bei PlaquePsoriasis zu einer signifikanten und klinisch relevanten Verbesserung gemessen am PASI-75-Score (d. h. eine Verbesserung des Psoriasis Area and Severity Index um $75 \%$ ) nach 16 Wochen, dem primären Endpunkt. Bei Patienten, die Apremilast erhalten hatten, war das Ansprechen von schwer zu behandelnden Körperbereichen wie den Nägeln und der Kopfhaut deutlich besser, was auch für den Juckreiz galt. Dies hat einen deutlichen Einfluss auf die Lebensqualität der Patienten sowie ihre persönliche Wahrnehmung der eigenen Krankheitslast.

Im Zuge des PALACE-Studienprogramms führte die Behandlung zu signifikanten und klinisch relevanten Verbesserungen der Symptome einer Psoriasis-Arthritis, wie Messungen des modifizierten ACR20-Ansprechens (d. h. eine 20-prozentige Verbesserung der Krankheitsaktivität gemäß den Kriterien des American College of Rheumatology) nach 16 Wochen, dem primären Endpunkt, zeigten. Bei Patienten, die Apremilast erhielten, wurden Verbesserungen bei typischen Manifestationen der Psoriasis-Arthritis festgestellt, wie zum Beispiel hinsichtlich geschwollener und schmerzempfindlicher Gelenke, Daktylitis- und Enthesitis-Manifestationen, der allgemeinen körperlichen Funktionsfähigkeit und der Lebensqualität.

\section{Die meisten Nebenwirkungen schwach oder moderat}

Im Verlauf dieser klinischen PhaseIII-Studien gehörten zu den am häufigsten beobachteten unerwünschten Ereignissen: Diarrhoe, Übelkeit, Infektion der oberen Atemwege, Spannungskopfschmerz und Kopfschmerz. Unerwünschte Ereignisse gastrointestinaler Natur traten meist in einem schwachen oder mäßigen Schweregrad auf. 0,3\% der Fälle von Diarrhoe und 0,3\% der Fälle von Übelkeit wurden als schwerwiegend empfunden. Diese Nebenwirkungen traten in der Regel innerhalb der ersten beiden Behandlungswochen auf und klangen generell im Verlauf von vier Wochen wieder ab. Insgesamt wurden die meisten Nebenwirkungen als schwach oder moderat eingestuft.

Die Entscheidung der Europäischen Kommission folgt auf die positive Stellungnahme des Ausschusses für Humanarzneimittel (CHMP) im November 2014. Apremilast wird in den kommenden Monaten in der Europäischen Union gemäß den lokalen Anforderungen auf den Markt gebracht.

\section{Quelle: \\ Pressemitteilung Celgene} Literatur auf Anfrage rheuma plus $2015 \cdot 14: 5$

DOI 10.1007/s12688-015-0024-4

Online publiziert: 12. Februar 2015

๑) Springer-Verlag Wien 2015 